Research Article

\title{
Nonlinear Dynamic Modelling of a Suspension Seat for Predicting the Vertical Seat Transmissibility
}

\author{
Weitan Yin $\mathbb{D}^{\mathbb{D}},{ }^{1}$ Juyue Ding $\mathbb{D}{ }^{2}$, and Yi Qiu $\mathbb{D}^{1}$ \\ ${ }^{1}$ Dynamics Group, Institute of Sound and Vibration Research, No. 1 University Rd, SO17 1BJ, Southampton, UK \\ ${ }^{2}$ School of Mechanics and Engineering Science, Shanghai University, Shanghai Institute of Applied Mathematics and Mechanics, \\ Shanghai 200444, China \\ Correspondence should be addressed to Yi Qiu; yi.qiu@soton.ac.uk
}

Received 24 July 2020; Accepted 14 August 2020; Published 6 December 2021

Academic Editor: Mauro Gaggero

Copyright ( $\odot 2021$ Weitan Yin et al. This is an open access article distributed under the Creative Commons Attribution License, which permits unrestricted use, distribution, and reproduction in any medium, provided the original work is properly cited.

\begin{abstract}
Suspension seats are widely used in heavy vehicles to reduce vibration transmitted to human body and promote ride comfort. Previous studies have shown that the dynamics of the suspension seat exhibits nonlinear behaviour with changed vibration magnitudes. Despite various linear seat models developed in the past, a nonlinear model of the suspension seat capturing the nonlinear dynamic behaviour of the seat suspension and cushion has not been developed for the prediction of the seat transmissibility. This paper proposes a nonlinear lumped parameter model of the suspension seat to predict the nonlinear dynamic response of the seat. The suspension seat model comprises of a nonlinear suspension submodel integrated with a nonlinear cushion submodel. The parameters of the submodels are determined by minimizing the error between the simulated and the measured transmissibility of the suspension mechanism and the force-deflection curve of the seat cushion, respectively. The model of the complete seat is then validated using the seat transmissibility measured with inert mass under vertical vibration excitation. The results show that the proposed suspension seat model can be used to predict the seat transmissibility with various excitation magnitudes.
\end{abstract}

\section{Introduction}

The drivers of trucks, busses, and off-road vehicles such as motor graders and excavators are often exposed to high magnitude Whole-Body Vibration (WBV) on work. Longterm exposure to WBV can have adverse effect on the health of the drivers of the heavy vehicles. For example, the high WBV exposures were found to be associated with the back pain-related absences of the heavy equipment vehicle mining operators [1]. Suspension seats are widely used in such vehicles to attenuate the vibration transmitted to the seated drivers. However, the suspension seat does not always provide attenuation of the vibration under every working condition [2-4], as depending on the working conditions of the vehicle, the vibration environment on different vehicles is different. This requires that the dynamic parameters of a suspension seat be designed or tuned to match with the dynamic characteristics of the vehicle, so as to optimize its vibration attenuation performance [5].
A suspension seat generally comprises two parts that play the most important role in the transmission of the WBV: the suspension mechanism and the seat cushion. The dynamic performance of the suspension seat depends on the dynamic properties of both parts. It was found in a laboratory study that when the magnitude of the vibration excitation was low, the suspension mechanism tended to act as if it was locked up by friction, and the seat transmissibility was mainly affected by the dynamic characteristics of the seat cushion, whereas when subjected to higher magnitudes of excitation, the dynamic performance of the suspension seat was dominated by the dynamics of the suspension rather than the seat cushion [6]. It was also found that the response of the suspension seat to the vertical vibration was nonlinear when the magnitude of excitation changed. With the increase of the excitation magnitude, the primary resonance frequency of the transmissibility of the suspension mechanism or the complete seat tended to decrease [6]. The seat cushion in modern vehicles is normally made of 
polyurethane foam, which also exhibits nonlinear behaviour that can be affected by the magnitudes of excitation [7]. Zhang \& Dupuis found that when the excitation magnitude increased, the equivalent stiffness of the foam tended to decrease at lower excitation magnitudes and then increase at higher magnitudes [8].

Apart from laboratory studies, the vibration transmission and dynamic response of suspension seats have also been studied with various analytical and numerical models. The modelling methods varied from the finite element method [9] to multibody method and lumped parameter method, and in recent years, researchers have made a lot of outstanding work $[10,11]$. Among different modelling methods available, lumped parameter technique has been widely applied, partly because it is relatively easy to apply, and a close form solution is often available. Qiu [5] established a systematic procedure for developing models of suspension seat by calibrating the submodels of the suspension mechanism and the seat cushion and connecting the two models as the seat model. Gunston et al. [12] developed a linear suspension seat model, whose parameters were taken from the measured dynamic properties of the suspension mechanism components. For example, the stiffness of the suspension spring and the damping were adopted as model parameters directly. Stein et al. [13] developed a lumped parameter model of the suspension seat where the damping of the suspension was modelled using a series combination of a linear spring and a linear damper. These linear models gave satisfactory predictions of the transmissibility of suspension seat at a fixed magnitude of excitation with a corresponding set of parameter values.

However, if a system exhibits evident nonlinear behaviour, such as the decrease of the resonance frequency of the suspension seat transmissibility when the excitation magnitudes increases, a linear model may require multiple sets of parameter values to fit well with the behaviour of the system under different conditions, which increases the cost of parameter optimization and simulation. On the other hand, nonlinear models may be more efficient and applicable when the system behaviour under different conditions is to be predicted with one set of parameter values. Various nonlinear lumped parameter seat models have been developed over the years which sought to capture the nonlinear behaviour of the suspension seat. In terms of nonlinear modelling of suspension mechanism, Gunston et al. [12] developed a suspension seat model using the so-called "Bouc-Wen model" $[14,15]$ which was verified using the vibration exposure value measured in a field study, while the prediction of the transmissibility of the suspension seat was not given. Zhou et al. [16] developed a model of the suspension seat consisting of nonlinear spring and damper of the suspension mechanism, and Zhao et al. [17] developed a nonlinear suspension seat with rubber spring. For both models, the predicted vibration exposure and seat transmissibility matched well with the measured counterparts, while the prediction under other conditions using the same set of parameter values was not reported in these studies. With regard to the nonlinear cushion modelling, Pattern and Pang [18] developed a lumped parameter model of a luxury car seat with nonlinear cushion stiffness (with algebraic fraction term) and damping (with absolute value term), which gave satisfactory prediction of the seat transmissibility. To further simulate the elastic and viscoelastic behaviour of the seat cushion, a nonlinear model of polyurethane foam taking both the elastic and viscoelastic stiffness into consideration was developed by White et al. [19]. Based on White's foam model, Kang [20] developed a nonlinear model of a railway vehicle seat to study the dynamic responses of the seat-occupant system, and Joshi et al. [21] developed a nonlinear seat-occupant model to predict the deflection shapes of the seat-occupant system. However, both predictions that Kang and Joshi et al. made using the model they developed lacked verification using the experimental data.

From the review of the previous studies, it is found that a model of suspension seat has not been reported, which covers the nonlinear dynamic behaviour of both the suspension mechanism and the seat cushion at different excitation magnitudes with just one set of parameter values and is capable of giving satisfactory and validated prediction of the transmissibility of the suspension seat under various excitations. The aim of the study presented in this paper is to develop a nonlinear lumped parameter model of a suspension seat consisting of both the nonlinear suspension submodel and the nonlinear cushion submodel that fulfils these requirements. In Section 2, the design and the results of the experimental measurement of the dynamic characteristics of the suspension mechanism and the seat cushion are presented. Section 3 presents the design and the calibration of the proposed nonlinear suspension seat model, and the model is validated by comparing the predicted seat transmissibility with the measured counterpart. General discussion of the developed nonlinear model is provided in Section 4 in which it is compared with a linear model, and the conclusion is summarised in Section 5.

\section{Experimental Study of Seat Transmissibility and Force-Deflection Relation of the Seat Cushion}

2.1. Measurement of the Vertical Transmissibility of the Suspension Seat with Inert Mass. A suspension seat with foam cushion and passive vertical suspension mechanism was used in this study (Figure 1). To study the nonlinear dynamics of the suspension mechanism and the complete seat, and to obtain experimental data for the calibration of the suspension seat model, the vertical transmissibilities of the suspension mechanism and the complete suspension seat loaded with a $60 \mathrm{~kg}$ inert mass were measured. The measurement was carried out on the $1 \mathrm{~m}$ vertical hydraulic simulator in the Institute of Sound and Vibration Research, University of Southampton. The vibration stimuli applied to the loaded suspension mechanism and the seat were three random vertical excitations with different magnitudes: 1.0, 1.5 , and $2.0 \mathrm{~ms}^{-2}$ r.m.s, respectively. The signals were acquired via $50 \mathrm{~Hz}$ anti-aliasing filters, and the sampling rate was 256 samples per second. To calculate the 


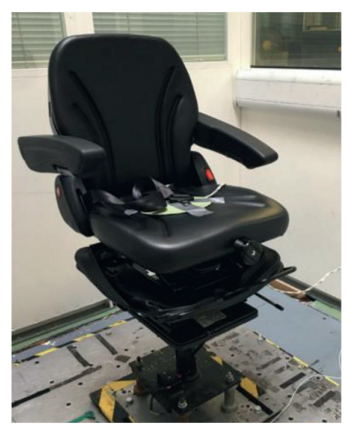

(a)

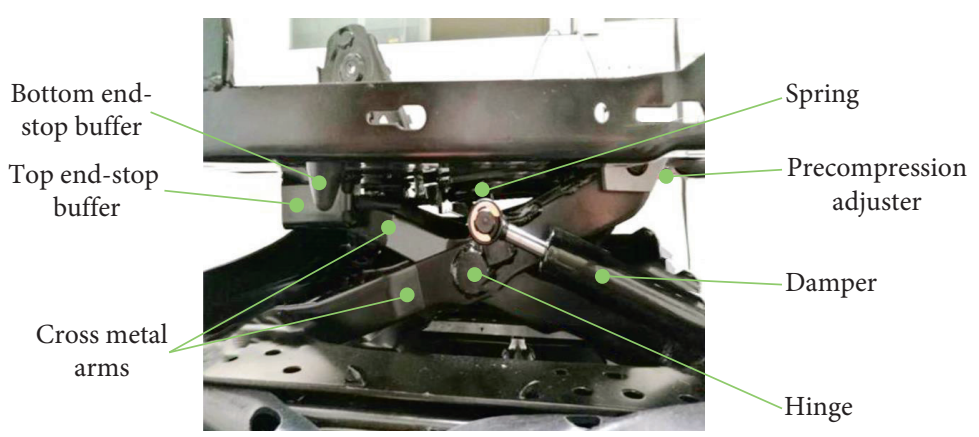

(b)

Figure 1: The suspension seat (a) and the structure of its suspension mechanism (b).

transmissibilities of the suspension mechanism and the complete seat $T(f)$, the vertical acceleration at the seat base $\left(a_{i}\right)$ was measured as the input acceleration, and the vertical acceleration on the suspension top plate or the complete seat pan $\left(a_{o}\right)$ was measured as the output acceleration. Then, the transmissibility of either the suspension mechanism or the complete seat is obtained using the cross-spectrum density method:

$$
T(f)=\frac{G_{i o}(f)}{G_{i}(f)},
$$

where $G_{i o}(f)$ is cross-spectral density of the input $\left(a_{i}\right)$ and output $\left(a_{o}\right)$ accelerations and $G_{i}(f)$ is the power spectral density of the input acceleration.

Figure 2 shows the moduli of the measured transmissibilities of the suspension mechanism and the complete seat. The primary resonances of both transmissibilities locate below $5 \mathrm{~Hz}$ with the associated moduli greater than unity, showing the amplification effect that the suspension seat had on the transmission of vibration at low frequency range. The moduli of transmissibilities at frequencies above $8 \mathrm{~Hz}$ are smaller than unity, showing an attenuation effect of the seat on the vibration in that frequency range. With the increase of the excitation magnitude, the primary resonances of the transmissibilities tend to decrease. It shows that the equivalent stiffness of the suspension mechanism as well as the complete seat exhibits a nonlinear softening behaviour with the increase of the excitation magnitude.

2.2. Measurement of the Force-Deflection Curves of the Seat Cushion. The quasi-static and dynamic behaviour of the seat cushion was characterised using the force-deflection curves in this study. The measurement of force-deflection curves of the seat cushion was carried out on the indenter rig in the Institute of Sound and Vibration Research, University of Southampton. The seat cushion was fixed on the vibrator of the indenter rig as shown in Figure 3. In the quasi-static test, the bottom of the seat cushion was fixed and the head of the indenter was forced to compress and decompress the top surface of the seat cushion in the vertical direction at a rate of $1.0 \mathrm{~mm} / \mathrm{s}$. During this process, the reaction force of the cushion and the displacement of the indenter head (i.e., the deflection of the cushion) were measured using the RDP

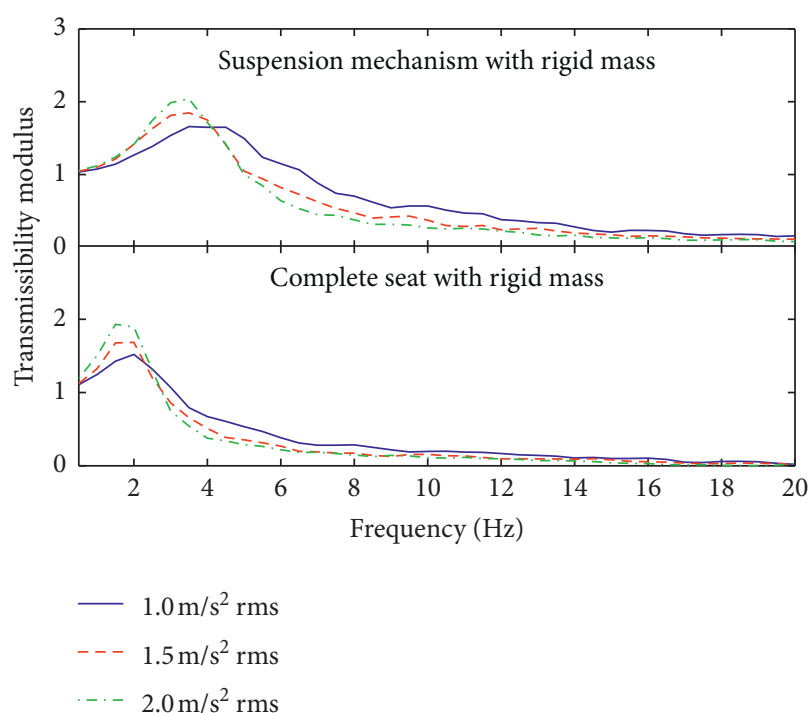

Figure 2: Measured transmissibilities of suspension mechanism and complete seat loaded with inert mass $(60 \mathrm{~kg})$ with excitation magnitudes of $1.0,1.5$, and $2.0 \mathrm{~ms}^{-2}$ r.m.s.

force transducer and RDP DWT displacement transducer, respectively. In the dynamic test, a preload of $600 \mathrm{~N}$ was applied to the cushion at a rate of $1.0 \mathrm{~mm} / \mathrm{s}$ using the indenter head. Then, three $5 \mathrm{~Hz}$ vertical sinusoidal excitations with magnitudes of $1.0,1.5$, and $2.0 \mathrm{~ms}^{-2}$ r.m.s were applied to the bottom of the cushion using the vibrator. The reaction force of the cushion and the displacement of the vibrator (i.e., the dynamic deflection of the cushion) were measured using the force transducer and a Celesco MT2A displacement transducer, respectively.

Figure 4 shows the measured quasi-static force-deflection hysteresis curve; i.e., for a certain value of the cushion reaction force, the loading and unloading paths give different deflections of the cushion. The gradient of the average curve (dotted line in Figure 4) of the loading and unloading parts of the hysteresis can be used to examine the nonlinear elastic property of the foam cushion [23]. The increase of the gradient indicates that the cushion was hardening with the increase of deflection during the quasi-static test.

The measured dynamic force-deflection curves are shown in Figure 5. To facilitate the comparison of the 


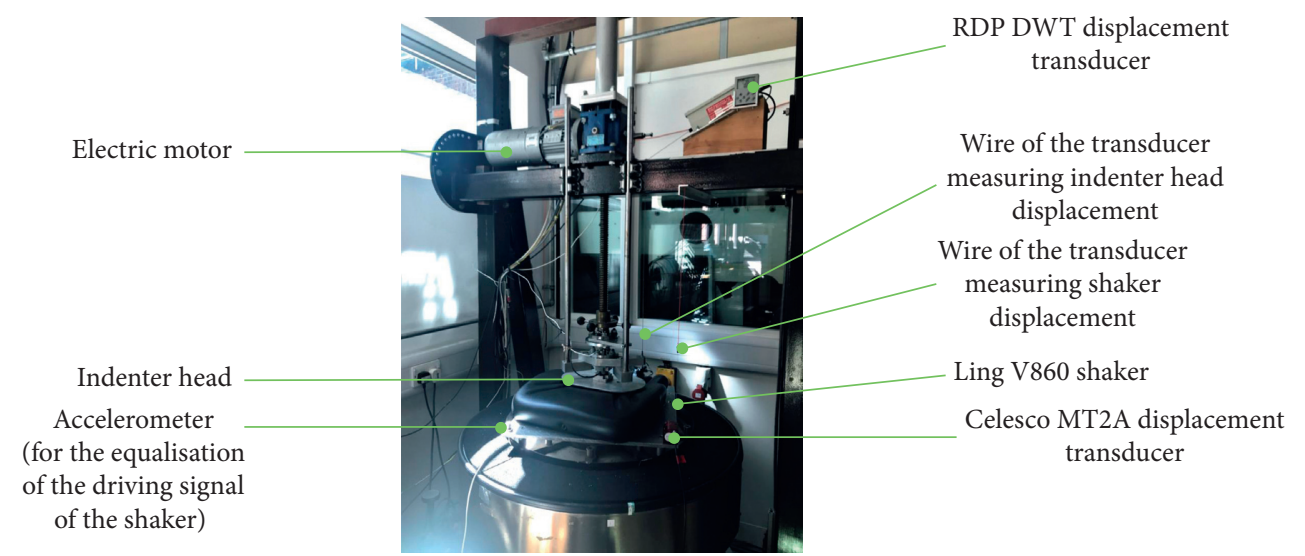

FIgURE 3: Test apparatus for the measurement of the force-deflection curves of the seat cushion.

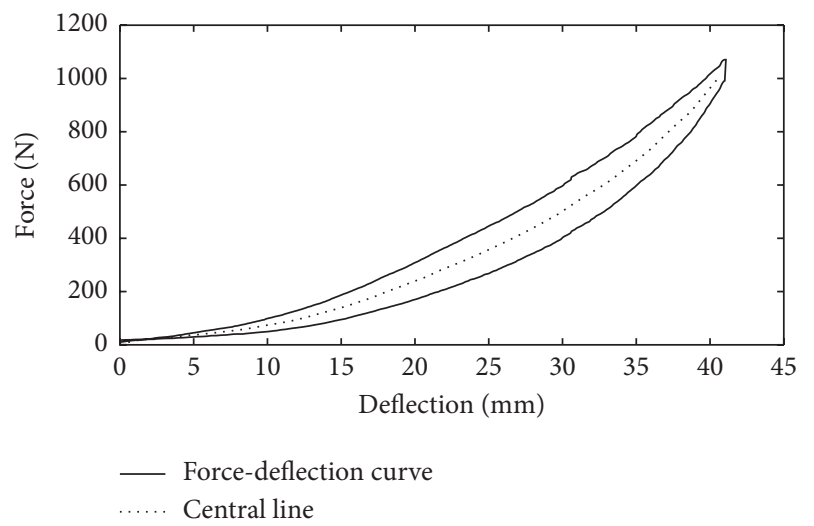

FIGURE 4: Quasi-static force-deflection curve of the cushion and the central line.

gradients of the curves, the dynamic force-deflection curves are translated by removing the static component (i.e., the values at the starting points of the curves) from the measured forces and deflections, so that their starting points all locate at the origin point $(0,0)$ (see Figures $5(\mathrm{a})$ and $5(\mathrm{~b})$ ).

Figure 5(a) shows that the gradients of dynamic forcedeflection curves were greater than those of quasi-static force-deflection curves, which indicates that the cushion is stiffer in dynamic conditions than in quasi-static conditions. Figure 5(b) shows that the gradients of the curves decreased when the excitation magnitude increased, which reveals the softening behaviour of the cushion subject to the increase of the magnitude of the excitation. Owing to the method of the calibration of the transducers, the dynamic deflections measured using the displacement transducer only revealed the relative dynamic displacements of the cushion surface above or below the static deflection, which was due to the application of the preload. Thus, to obtain the total deflection of the cushion relative to the unloaded state, the static deflection of the cushion should be added to the measured dynamic deflection. The static deflection of the cushion is estimated to be $0.033 \mathrm{~m}$ when the preload applied on the cushion is $600 \mathrm{~N}$ according to the central line of the quasi-static force-deflection curve (Figure 4). The forcedeflection curves with the actual deflection are shown in
Figure 5(c) and will be used for calibration of the cushion submodel in the next stage. It shows that, without translation, the gradients of these original curves are difficult to compare.

\section{Nonlinear Suspension Seat Model}

3.1. Modelling Method. As both the suspension mechanism and the seat cushion have nonlinear response to the vibration excitation, it is desired to develop a suspension seat model that consists of both nonlinear suspension and cushion submodels based on the results of the measurement. In this study, the submodel of the suspension mechanism was developed using the "Bouc-Wen method" whereas the cushion submodel was developed with polynomial nonlinear stiffness and viscoelastic terms.

While the spring and damper of the suspension mechanism can be treated as a combination of linear spring and damper [22], the "Bouc-Wen method" can be added to that linear combination to model the nonlinear forces in the suspension mechanism, such as the friction. Assuming that the displacement of the suspension mechanism relative to the seat base is $y$, the total reaction force of the loaded suspension mechanism $F_{\text {suspension }}$ can be written as follows:

$$
F_{\text {suspension }}=k_{s} y(t)+c_{s} \dot{y}(t)+F_{\text {Bouc-Wen }} \text {, }
$$

where the linear stiffness and damping of the suspension mechanism are represented by $k_{s}$ and $c_{s}$, respectively, and the "Bouc-Wen force" $F_{\text {Bouc-Wen }}$ is determined as follows [12]:

$$
\dot{F}_{\text {Bouc-Wen }}=\left(k-k_{s}\right) \dot{y}-\gamma|\dot{y}| F_{\text {Bouc-Wen }}-\beta \dot{y}\left|F_{\text {Bouc-Wen }}\right|,
$$

where $k, \beta$, and $\gamma$ are three parameters determining the nonlinearity of the "Bouc-Wen force."

On the other hand, the cushion nonlinear behaviour owes much to the cell structure of the polyurethane foam and the air inside the cells that caused the cushion to exhibit nonlinear elastic property [18]. A modelling method taking this into account was applied to develop the cushion model in this study. Under dynamic conditions, the polyurethane 

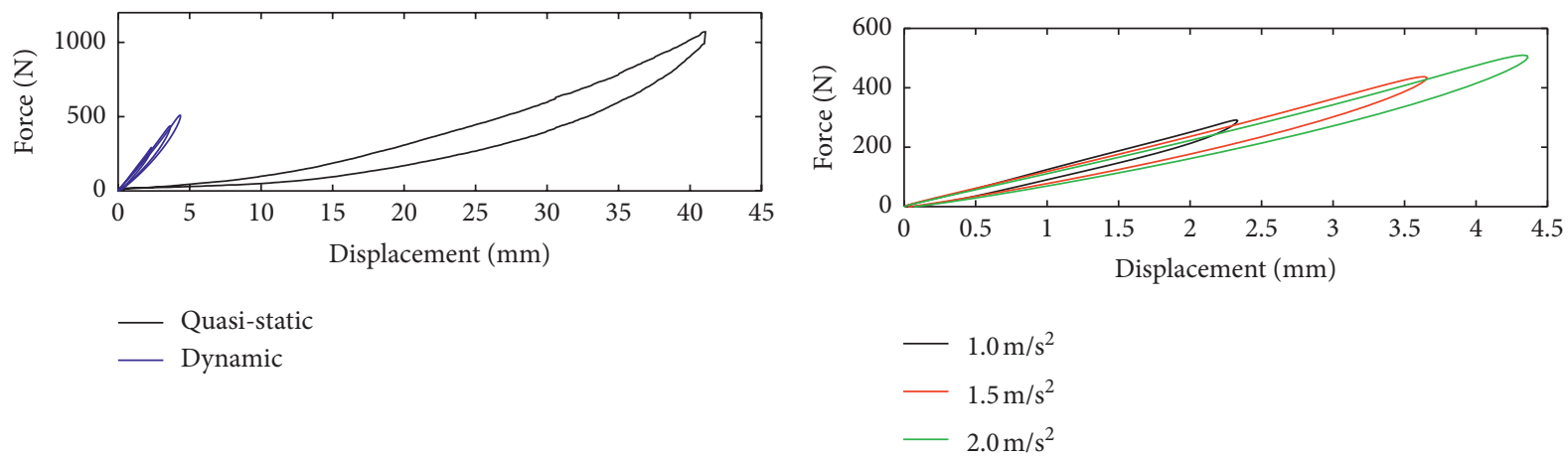

(a)

(b)

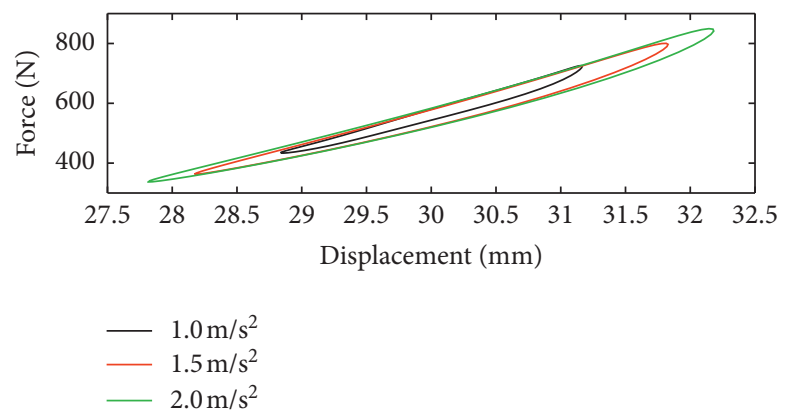

(c)

FIgURe 5: (a) A comparison between quasi-static and dynamic force-deflection curves, (b) translated dynamic force-deflection curves with different excitation magnitudes, and (c) dynamic force-deflection curves with the actual deflection.

foam will generate elastic, viscous, and viscoelastic reaction forces [23]. All these three types of forces are included in the model of the seat cushion to reflect the nonlinear dynamic behaviour of the cushion. When the cushion is compressed, the reaction force $F_{\text {cushion }}$ from the cushion is derived using the following equation:

$$
\begin{aligned}
F_{\text {cushion }}= & c \dot{z}(t)+k_{1} z(t)+k_{3} z(t)^{3}+k_{5} z(t)^{5} \\
& +\int_{0}^{t} \Gamma(t-\tau) z(t) d \tau
\end{aligned}
$$

where $z$ is the deflection of the cushion (i.e., the displacement of the cushion surface relative to the cushion base) and $c, k_{1}, k_{3}$, and $k_{5}$ stand for the viscous damping and nonlinear stiffness parameters, respectively. The convolution of displacement and relaxation kernel $\Gamma$ is used to reveal the nature of the history-depending hysteresis of the cushion force-deflection curves. The relaxation kernel $\Gamma$ is defined as a summation of two exponential terms:

$$
\Gamma(t-\tau)=a_{1} e^{-\alpha_{1}(t-\tau)}+a_{2} e^{-\alpha_{2}(t-\tau)},
$$

where $a_{1}, a_{2}$ and $\alpha_{1}, \alpha_{2}$ are viscoelastic parameters [21]. By calibrating the cushion model described in equation (4) using the measured dynamic force-deflection curve, the elastic, viscous, and viscoelastic parameters of the cushion can be obtained. Then, the cushion submodel was connected with the suspension mechanism model to build up the complete suspension seat model.
3.2. Description of the Model. The complete suspension seat model loaded with an inert mass is shown schematically in Figure 6. The motion of the seat base (i.e., the excitation) is represented by $x_{0}$. The sprung mass of the suspension mechanism is represented by $m_{s}$, which is estimated to be $4.6 \mathrm{~kg}$. The stiffness and damping of the suspension mechanism are represented by $k_{s}$ and $c_{s}$, respectively. $F_{\text {Bouc-Wen }}$ is the Bouc-Wen force. The inert mass loaded on the seat is represented by $m_{0}(60 \mathrm{~kg})$. The cushion is divided into the unsprung mass $m_{c 1}$ and the sprung mass $m_{c 2}$, and both masses are estimated to be $10 \mathrm{~kg}$. The cushion unsprung mass $m_{c 1}$ moves together with $m_{s}$, while $m_{c 2}$ moves together with the inert mass $m_{0}$. The elastic stiffness of the cushion is represented by a nonlinear polynomial with the first-, third-, and fifth-order coefficients $k_{1}, k_{3}$, and $k_{5}$, and $c$ represents the viscous damping of cushion. The viscoelastic force $F_{\mathrm{ve}}$ is represented by the convolution term (see equation (4)).

Since the seat was excited after being loaded with the inert mass on the seat pan, the static deflection of the cushion $d$ due to the static preload is also included in the equations; thus, the dynamic deflection of the cushion is expressed by the term $\left(x_{2}-x_{1}-d\right)$. In Figure 4 , the static deflection of the cushion with a preload of $600 \mathrm{~N}$ was approximately $0.033 \mathrm{~m}$. However, due to the creep behaviour of the cushion foam [19], i.e., the deflection tended to gradually decrease with time when a constant preload was applied, the difference between the quasi-static loading of the cushion during the measurement of the force-deflection relationship and the loading of the inert mass during the measurement of the seat transmissibility may lead to minor 


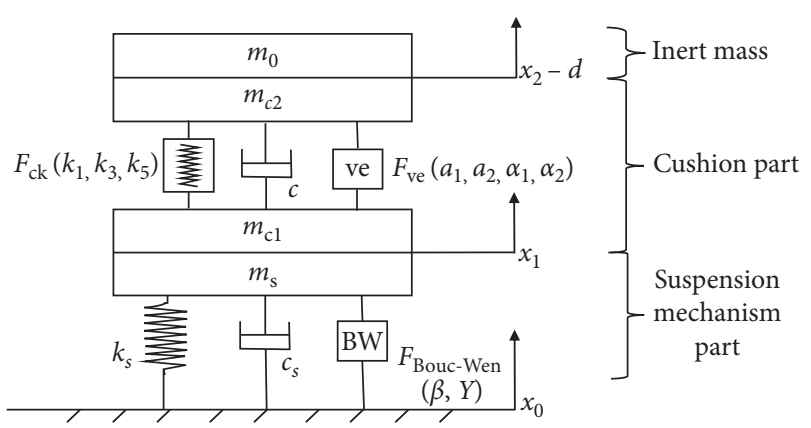

FIGURE 6: Schematic diagram of the complete seat model loaded with inert mass.

difference between the deflection of the cushion in these two tests. Hence, in this model, the value of the deflection $d$ was determined by doing calibration with a specific constraint applied $(0.025<d<0.035)$ along with the rest of the model parameters. The equations of motion of the complete seat loaded with inert mass are derived as follows:

$$
\begin{gathered}
\left(m_{c 2}+m_{0}\right) \ddot{x}_{2}+F_{\mathrm{ck}}+c\left(\dot{x}_{2}-\ddot{x}_{1}\right)+F_{\mathrm{ve}}=-\left(m_{c 2}+m_{0}\right) g \\
\left(m_{s}+m_{c 1}\right) \ddot{x}_{2}+c_{s}\left(\dot{x}_{1}-\dot{x}_{0}\right)+k_{s}\left(x_{1}-x_{0}\right)-F_{\mathrm{ck}} \\
\quad-c\left(\dot{x}_{2}-\dot{x}_{1}\right)-F_{\mathrm{ve}}+F_{\text {Bouc-Wen }}=0
\end{gathered}
$$

where the cushion elastic force $F_{c k}$ is defined as follows:

$$
F_{\mathrm{ck}}=k_{1}\left(x_{2}-x_{1}-d\right)+k_{3}\left(x_{2}-x_{1}-d\right)^{3}+k_{5}\left(x_{2}-x_{1}-d\right)^{5} \text {, }
$$

and the cushion viscoelastic force $F_{\mathrm{ve}}$ is defined as follows:

$$
F_{\mathrm{ve}}=\int_{0}^{t}\left[a_{1} e^{-\alpha_{1}(t-\tau)}+a_{2} e^{-\alpha_{2}(t-\tau)}\right]\left(x_{2}-x_{1}-d\right) d \tau,
$$

and the "Bouc-Wen force" $F_{\mathrm{BW}}$ in this complete seat model is defined as follows:

$$
\begin{aligned}
\dot{F}_{\text {Bouc-Wen }}= & \left(k-k_{s}\right)\left(\dot{x}_{1}-\dot{x}_{0}\right)-\gamma\left|\dot{x}_{1}-\dot{x}_{0}\right| F_{\text {Bouc-Wen }} \\
& -\beta\left(\dot{x}_{1}-\dot{x}_{0}\right)\left|F_{\text {Bouc-Wen }}\right| .
\end{aligned}
$$

3.3. Calibration of the Submodels and Validation of the Complete Seat Model. The complete seat model was calibrated in the following order: first, the nonlinear suspension mechanism model was calibrated by fitting the measured acceleration on the suspension top plate in the time domain, such that the parameters of the suspension mechanism model $\left(k, k_{s}, k_{c}, \beta\right.$, and $\left.\gamma\right)$ were determined. Then, the cushion submodel was calibrated using the measured actual dynamic force-deflection curves of the cushion (shown in Figure 5(c)), and the parameters of the cushion model $\left(k_{1}, k_{3}, k_{5}, c, a_{1}, a_{2}, \alpha_{1}, \alpha_{2}\right.$, and $\left.d\right)$ were decided. Finally, the calibrated suspension submodel and the cushion submodel were connected as the complete seat model (schematically shown in Figure 6). The complete seat model was used to predict the acceleration on the seat pan loaded with inert mass and the seat transmissibility with different excitation magnitudes, and the results were compared with their counterpart measured using the suspension seat supporting the inert mass to validate the seat model.

A linear lumped parameter model would be required to be recalibrated to obtain new sets of parameters to give satisfactory predictions of seat dynamic behaviour under various excitations due to its nonlinearity. To obtain one set of parameters of the nonlinear model that is capable of predicting the seat dynamic performance under different excitation magnitudes, each submodel was calibrated by minimizing the total error $\left(E_{\text {total }}\right)$, which was defined as the sum of root mean square differences between the predicted and measured data under all the three excitation magnitudes (1.0, 1.5 , and $2.0 \mathrm{~ms}^{-2}$ r.m.s.):

$$
E_{\text {total }}=\sum_{i=1}^{3} \sum_{j=1}^{N} \frac{1}{N} \sqrt{\left(p_{i j}-m_{i j}\right)^{2}},
$$

where the subscript $i(i=1,2,3)$ represents the three tests with different excitation magnitudes, while the subscript $j$ $(j=1,2,3, \ldots, N)$ represents the individual data sample under each test condition. Each of the predicted data samples is represented by $p_{i j}$, and the corresponding measured data sample is represented by $m_{i j}$, respectively. Figure 7 shows a comparison between measured and predicted output acceleration on the suspension top plate using the suspension submodel, and a good agreement was achieved. Figure 8 shows that the predicted suspension mechanism transmissibilities (frequency domain) also fit with the corresponding measured data well.

Figure 9 shows a comparison between the measured dynamic force-deflection curves of the cushion and those simulated using the cushion submodel with a preload of $600 \mathrm{~N}$ and with different excitation magnitudes. The simulated curves generally fit with the test results well.

The complete seat model parameters obtained after calibration are listed in Table 1. Finally, a comparison between the measured and predicted accelerations on the seat pan when the seat was loaded with inert mass is made in Figure 10, in which a reasonably good agreement is observed. The measured and predicted transmissibilities of the complete seat loaded with inert mass are shown in Figure 11. It shows that the prediction generally fits with the test data, but some discrepancies are found in terms of transmissibility modulus in the vicinity of the primary resonance.

\section{Discussion}

4.1. Dynamic Characteristics of the Seat Components. The resonance of the transmissibility of the suspension mechanism in this study tended to decrease with the increase of the magnitude of the vertical excitation, which indicates the nonlinearity of the suspension mechanism similar to that presented by Qiu [6]. The quasi-static force-deflection curve of the cushion shows a hysteresis loop, which is in accordance with many previous studies (e.g., $[19,23,24])$. The 


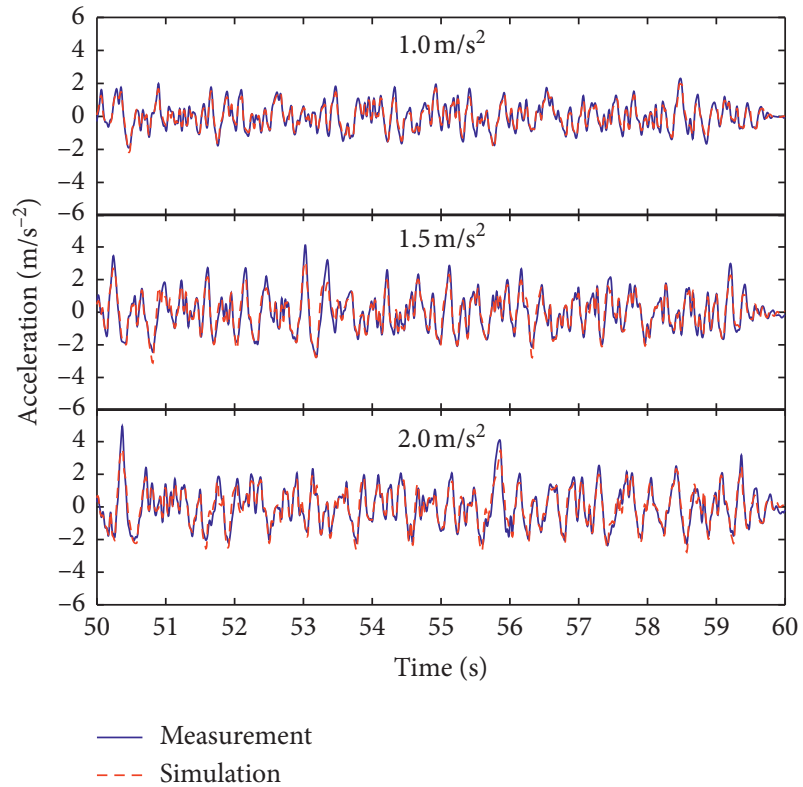

Figure 7: A comparison between the measured and the predicted acceleration on the suspension top plate in the last 10 seconds with different excitation magnitudes (1.0, 1.5, and $2.0 \mathrm{~ms}^{-2}$ r.m.s.).

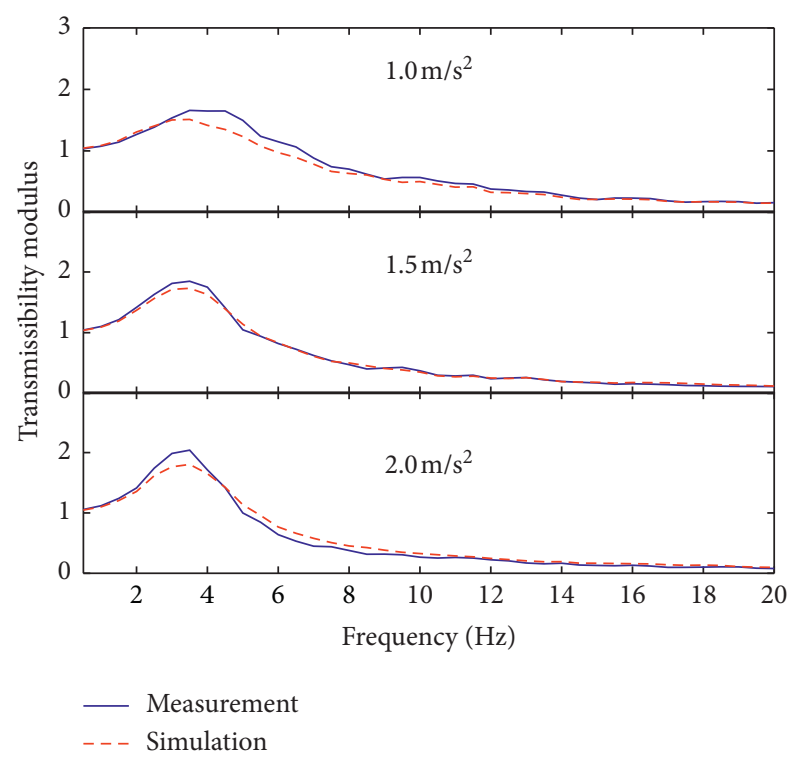

Figure 8: A comparison between the measured and predicted transmissibility of suspension mechanism with different excitation magnitudes (1.0, 1.5, and $2.0 \mathrm{~ms}^{-2}$ r.m.s.).

dynamic force-deflection curves have greater gradients than that of the quasi-static force-deflection curve, showing the hardening of the cushion stiffness under dynamic conditions. The dynamic force-deflection curves in this test show that the dynamic stiffness of the seat cushion is affected by the magnitude of excitation, indicating the nonlinearity of the cushion behaviour. Both seat components hence processed nonlinearity subject to the excitation magnitude.
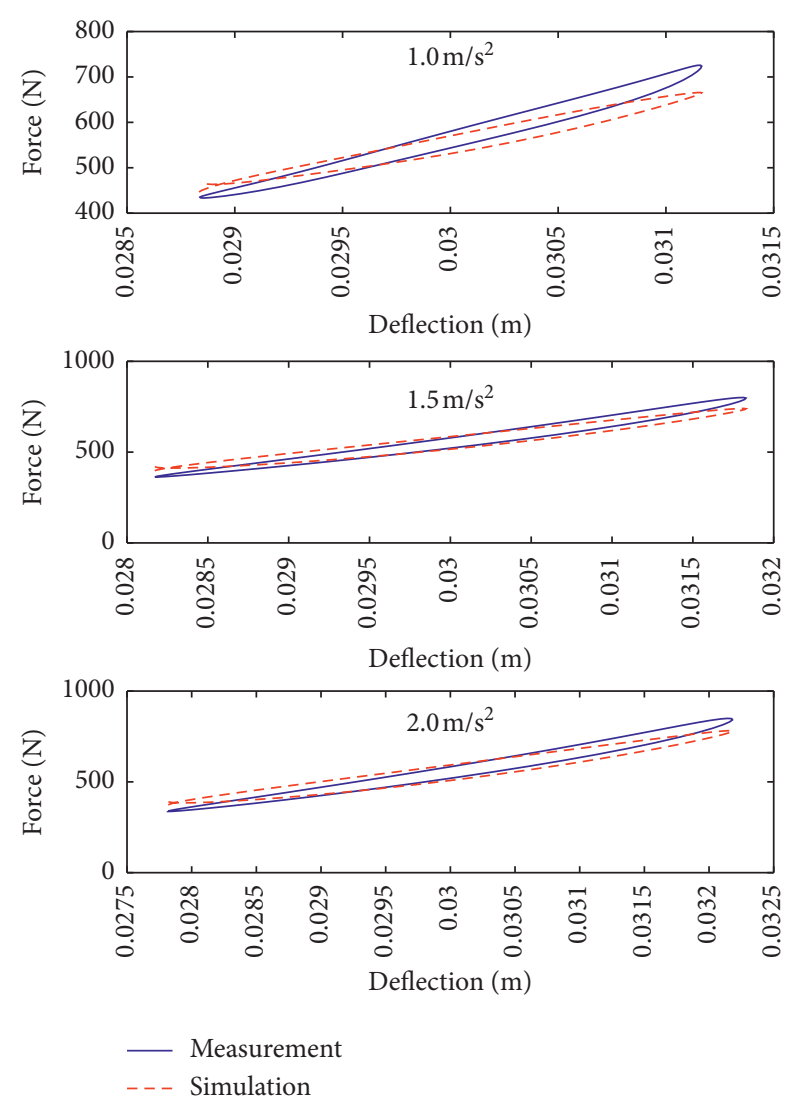

FIgURE 9: A comparison between the simulated dynamic forcedeflection curve using the calibrated cushion submodel and measured dynamic force-deflection curve with three excitation magnitudes (1.0, 1.5 , and $2.0 \mathrm{~ms}^{-2}$ r.m.s.).

4.2. Linear and Nonlinear Modelling Methods. In the past, many lumped parameter suspension seat models were developed based on the linear assumption. The linear models reflect the dynamic property of the seat by means of stiffness and damping, which facilitates the understanding of seat dynamics and the design of the seat. In this study, a linear model of the suspension mechanism was also developed using the same way as Stein et al. [13] (see Figure 12).

The equivalent stiffness of the suspension mechanism was modelled using a spring element with the stiffness of $k_{s}$, and the damping was modelled using a series combination of a damper $(c)$ and a spring $(k)$. Then, the equations of motion of this system can be written as follows:

$$
\begin{aligned}
\left(m_{s}+m_{0}\right) \ddot{x}_{1}+c\left(\dot{x}_{1}-\dot{x}_{m}\right)+k\left(x_{1}-x_{m}\right) & =0, \\
c\left(\dot{x}_{m}-\dot{x}_{0}\right)+k\left(x_{m}-x_{0}\right) & =0 .
\end{aligned}
$$

Equations (12) and (13) can be reorganised as follows:

$$
\mathbf{M} \ddot{x}+\mathbf{C} \dot{x}+\mathbf{K x}=\left[\begin{array}{ll}
k_{s} & k
\end{array}\right]^{T} x_{0},
$$

where $\mathbf{M}=\left[\begin{array}{cc}m_{s}+m_{0} & 0 \\ 0 & 0\end{array}\right], \mathbf{C}=\left[\begin{array}{cc}c & -c \\ -c & c\end{array}\right], \mathbf{K}=\left[\begin{array}{cc}k_{s} & 0 \\ 0 & k_{s}\end{array}\right]$, and $\mathbf{x}=\left[x_{1} x_{m}\right]^{T}$. With this linear model, the transmissibility of the suspension mechanism $T$ can be derived as follows: 
TABLE 1: Calibrated parameter values of the complete seat model.

\begin{tabular}{|c|c|c|c|c|c|}
\hline \multirow{2}{*}{$\begin{array}{l}\text { Submodel } \\
\text { Suspension mechanism }\end{array}$} & \multicolumn{5}{|c|}{ Parameters and values } \\
\hline & $\begin{array}{l}k_{s}(\mathrm{~N} / \mathrm{m}) \\
32300.36\end{array}$ & $\begin{array}{c}c_{s}(\mathrm{Ns} / \mathrm{m}) \\
514.11\end{array}$ & $\begin{array}{c}k(\mathrm{~N} / \mathrm{m}) \\
242962.25\end{array}$ & $\begin{array}{c}\beta \\
-121.69 \\
\end{array}$ & $\begin{array}{c}\gamma \\
8449.14 \\
\end{array}$ \\
\hline Cushion & $\begin{array}{c}k_{1}(\mathrm{~N} / \mathrm{m}) \\
100.04 \\
a_{1}\left(\mathrm{~N} / \mathrm{m}^{2}\right) \\
79811.91\end{array}$ & $\begin{array}{c}k_{3}(\mathrm{~N} / \mathrm{m}) \\
-8255.13 \\
\alpha_{1}(1 / \mathrm{s}) \\
0.45\end{array}$ & $\begin{array}{l}k_{5}(\mathrm{~N} / \mathrm{m}) \\
2.23 E+10 \\
a_{2}\left(\mathrm{~N} / \mathrm{m}^{2}\right) \\
-79676.07\end{array}$ & $\begin{array}{c}c(\mathrm{Ns} / \mathrm{m}) \\
677.60 \\
\alpha_{2}(1 / \mathrm{s}) \\
1.00\end{array}$ & $\begin{array}{c}d(\mathrm{~m}) \\
0.03\end{array}$ \\
\hline
\end{tabular}

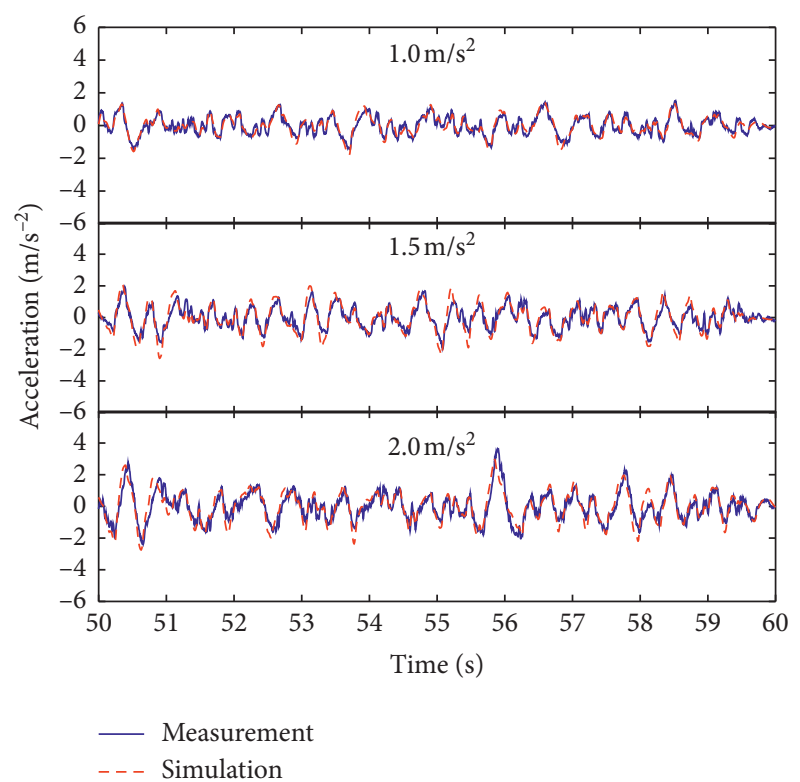

Figure 10: A comparison between the measured and the predicted acceleration on the seat pan with the complete seat loaded with inert mass within 10 seconds with different excitation magnitudes $\left(1.0,1.5\right.$, and $2.0 \mathrm{~ms}^{-2}$ r.m.s.).

$$
T(\omega)=\frac{X(\omega)}{X_{0}(\omega)}=\frac{\left[\begin{array}{ll}
k_{s} & k
\end{array}\right]^{T}}{\left[-\omega^{2} \mathbf{M}+i \omega \mathbf{C}+\mathbf{K}\right]}
$$

By minimizing the total error between the predicted transmissibilities (using equation (15)) and the measured counterparts under three excitations at the same time, the values of one set of parameters of this linear suspension mechanism model under three excitation magnitudes were determined and the comparison between the results of the measurement and simulation is shown in Figure 13. The predicted transmissibility fitted well with the measured value when the magnitudes of excitation were 1.5 and $2.0 \mathrm{~ms}^{-2}$ r.m.s, while at $1.0 \mathrm{~ms}^{-2}$ r.m.s, the discrepancy between the measured and simulated values of the resonance frequency and the modulus of the transmissibility in the vicinity of the resonance was greater compared to that with nonlinear Bouc-Wen model of the suspension mechanism (Figure 8). As the nonlinearity of the dynamic behaviour of the suspension mechanism was revealed by the change of the resonance frequency and the associated modulus of the

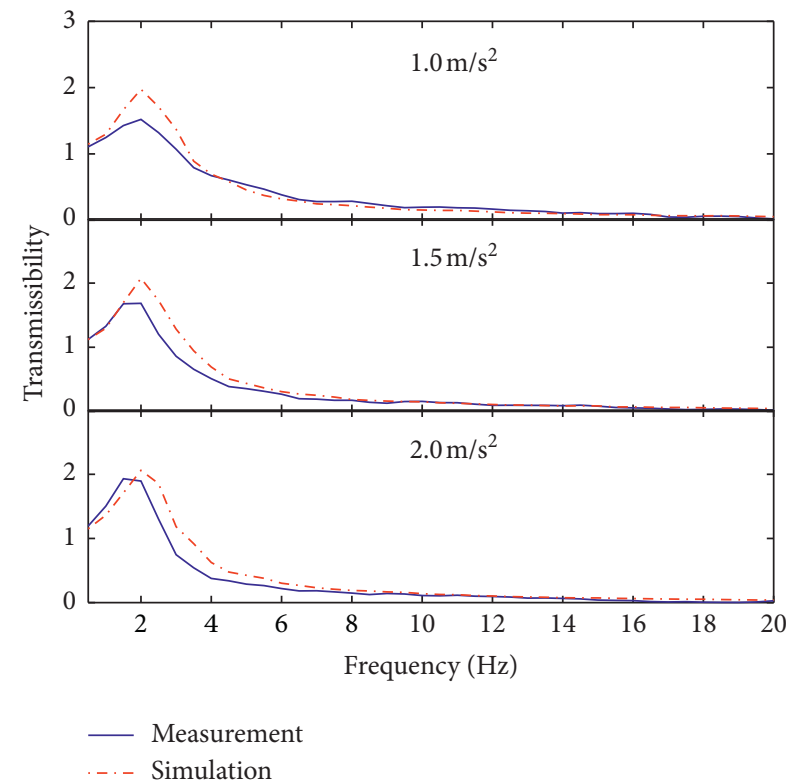

Figure 11: A comparison between the measured and predicted transmissibility of complete seat with different excitation magnitudes $\left(1.0,1.5\right.$, and $2.0 \mathrm{~ms}^{-2}$ r.m.s.).

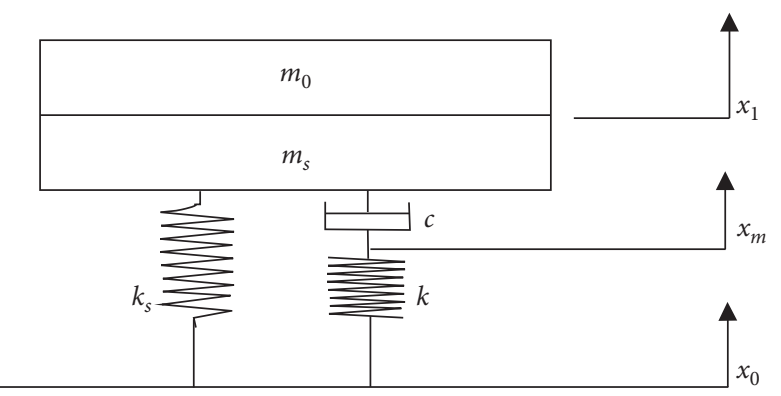

FIGURE 12: Schematic diagram of the linear suspension mechanism model.

transmissibility, the linear model failed to cover the nonlinear behaviour under all conditions. The nonlinear model of the suspension mechanism gave satisfactory predictions in a wider range using just one set of model parameters.

The disadvantage of the nonlinear models is that physical meaning of some of the model parameters is less clear than the linear models, such as the parameters $\beta$ and $\gamma$ of the "Bouc-Wen" model. This makes nonlinear models less practical for the optimization of real seat parameters 


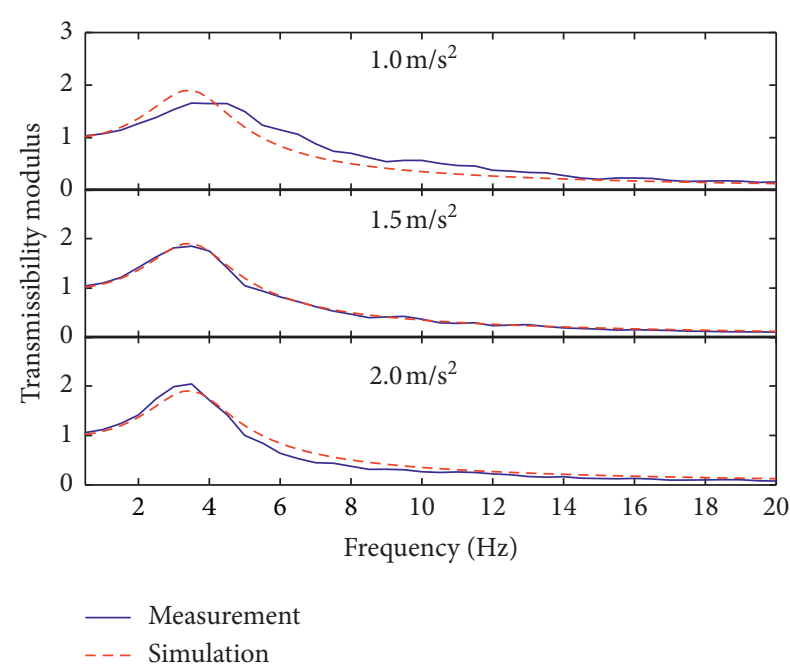

FIGURE 13: The prediction of the transmissibility of suspension mechanism using the linear suspension mechanism model.

compared to the linear models. However, in cases where a description of the general complete seat behaviour is sufficient, such as studying the interaction between the complete seat and the seated occupant, or predicting the WholeBody Vibration exposure of seated occupant in the vehicle, nonlinear models can still find their application [12].

\subsection{Measurement of the Parameters for the Calibration of the} Model. The suspension submodel can give a reasonably good prediction of the suspension transmissibility. However, some discrepancies existed between the measured and simulated dynamic force-deflection curves as well as the complete seat transmissibility. The reason may be that the loading and unloading speed in the quasi-static test of this study was $1 \mathrm{~mm} / \mathrm{s}$ due to the limitation of test apparatus, which is quite faster than that in the quasi-static tests reported in previous studies and may not be a real quasi-static state $[19,23]$. Zhang stated that, with the increase of loading speed, the quasi-static stiffness of the cushion also increased [24]. Thus, the loading speed in this study may affect the measurement of the quasi-static force-deflection curve and then have an impact on the static cushion deflection estimation (the value of $d$ ) and finally affect the calibration of the cushion model. Furthermore, in the dynamic forcedeflection curve measurement in this study, the excitations applied to the cushion are sinusoidal signals with one fixed frequency $(5 \mathrm{~Hz})$. However, it is reported that the dynamic stiffness of the cushion tended to increase with the increase of the frequency of the excitation $[7,25]$. The dynamic forcedeflection curves measured in this paper with single frequency sinusoids may not have completely reflected the dynamic behaviour of the cushion.

For the further study in the next stage, the test procedure of measuring force-deflection curves needs to be improved to obtain more accurate static force-deflection curve. The dynamic force-deflection curves under excitations of different frequencies will be measured and applied in the model calibration, so as to achieve a better prediction of the seat transmissibility.

\section{Conclusion}

A nonlinear lumped parameter model of a suspension seat was developed. The complete seat model consisted of nonlinear submodels of the suspension mechanism and the seat cushion. The submodels were calibrated using the experimental data measured with the suspension mechanism and the seat cushion, respectively. The integrated complete seat model gave satisfactory prediction of the seat transmissibility with different excitation magnitudes, indicating that it can be used to predict the vertical transmissibility of the suspension seat.

\section{Data Availability}

Data are available from the corresponding author upon request.

\section{Conflicts of Interest}

The authors declare that they have no conflicts of interest.

\section{Acknowledgments}

Thanks are due to Li Haisheng for assistance with the experiments and the analysis of the data.

\section{References}

[1] L. H. Barrero, M. Cifuentes, A. C. Rodríguez et al., "Wholebody vibration and back pain-related work absence among heavy equipment vehicle mining operators," Occupational and Environmental Medicine, vol. 76, no. 8, pp. 554-559, 2019.

[2] S. A. Adam and N. A. A. Jalil, "Vertical suspension seat transmissibility and SEAT values for seated person exposed to whole-body vibration in agricultural tractor preliminary study," Procedia Engineering, vol. 170, pp. 435-442, 2017.

[3] P. W. Johnson, J. Ibbotson-Brown, S. Menocal, and J. Parison, "Comparison of whole-body vibration exposures when operating a city bus with an active, passive and static suspension bus seat," Proceedings of the Human Factors and Ergonomics Society Annual Meeting, vol. 63, no. 1, pp. 1052-1056, 2019.

[4] J. H. Kim, L. S. Marin, and J. T. Dennerlein, "Evaluation of commercially available seat suspensions to reduce whole body vibration exposures in mining heavy equipment vehicle operators," Applied Ergonomics, vol. 71, pp. 78-86, 2018.

[5] Y. Qiu, "A procedure for developing a generic model of a suspenison seat with occupant for predicting seat transmissibility," in Proceedings of the 47th United Kingdom Conference on Human Responses to Vibration, ISVR, University of Southampton, Southampton, England, May 2012.

[6] Y. Qiu, "Dynamic characteristics of a suspension seat determined in laboratory study," Journal of Ergonomics, vol. 7, no. 6, p. 2, 2017.

[7] S. Tufano and M. J. Griffin, "Nonlinearity in the vertical transmissibility of seating: the role of the human body apparent mass and seat dynamic stiffness," Vehicle System Dynamics, vol. 51, no. 1, pp. 122-138, 2013.

[8] L. Zhang and R. Dupuis, "Measurement and identification of dynamic properties of flexible polyurethane foam," Journal of Vibration and Control, vol. 17, no. 4, pp. 517-526, 2011.

[9] R. Huang, S. Zheng, Z. Liu, and T. Y. Ng, "Recent advances of the constitutive models of smart materials-hydrogels and 
shape memory polymers," International Journal of Applied Mechanics, vol. 12, no. 2, Article ID 2050014, 2020.

[10] J. Cheng, "Analyzing the factors influencing the choice of the government on leasing different types of land uses: evidence from Shanghai of China," Land Use Policy, vol. 90, no. 90, Article ID 104303, 2020.

[11] J. Cheng, "Data analysis of the factors influencing the industrial land leasing in shanghai based on mathematical models," Mathematical Problems in Engineering, vol. 2020, Article ID 9346863, 11 pages, 2020.

[12] T. Gunston, J. Rebelle, and M. Griffin, “A comparison of two methods of simulating seat suspension dynamic performance," Journal of Sound and Vibration, vol. 278, no. 1-2, pp. 117-134, 2004.

[13] G. Stein, P. Múčka, T. P. Gunston, and S. Badura, "Modelling and simulation of locomotive driver's seat vertical suspension vibration isolation system," International Journal of Industrial Ergonomics, vol. 38, no. 5-6, pp. 384-395, 2008.

[14] R. Bouc, "Forced vibrations of mechanical systems with hysteresis," in Proceedings. of the Fourth Conference on Nonlinear Oscillations, Prague, Czechia, September 1967.

[15] Y.-K. Wen, "Method for random vibration of hysteretic systems," Journal of the Engineering Mechanics Division, vol. 102, no. 2, pp. 249-263, 1976.

[16] C. Zhou, L. Zhao, Y. Yu, F. Yang, and S. Wang, "Kinetodynamic model and simplified model of X-type seat system with an integrated spring damper," Shock and Vibration, vol. 2018, Article ID 4962436, 10 pages, 2018.

[17] L. Zhao, Y. Yu, C. Zhou, and F. Yang, "Modelling and validation of a seat suspension with rubber spring for off-road vehicles," Journal of Vibration and Control, vol. 24, no. 18, pp. 4110-4121, 2018.

[18] W. N. Patten and J. Pang, "Validation of a nonlinear automotive seat cushion vibration model," Vehicle System Dynamics, vol. 30, no. 1, pp. 55-68, 1998.

[19] S. W. White, S. K. Kim, A. K. Bajaj, P. Davies, D. K. Showers, and P. E. Liedtke, "Experimental techniques and identification of nonlinear and viscoelastic properties of flexible polyurethane foam," Nonlinear Dynamics, vol. 22, no. 3, pp. 281-313, 2000.

[20] J. Kang, "Human body vibration analysis under consideration of seat dynamic characteristics," Journal of the Korea Academia-Industrial Cooperation Society, vol. 13, no. 12, pp. 5689-5695, 2012.

[21] G. Joshi, A. K. Bajaj, and P. Davies, "Whole-body vibratory response study using a nonlinear multi-body model of seatoccupant system with viscoelastic flexible polyurethane foam," Industrial Health, vol. 48, no. 5, pp. 663-674, 2010.

[22] F. Shahzad and Y. Qiu, "A nonlinear multi-body dynamic model of a suspension seat with occupant correlated with experimental results," in Proceedings of the 47th United Kingdom Conference on Human Responses to Vibration, ISVR, University of Southampton, Southampton, England, September 2012.

[23] R. Ippili, P. Davies, A. K. Bajaj, and L. Hagenmeyer, "Nonlinear multi-body dynamic modeling of seat-occupant system with polyurethane seat and H-point prediction," International Journal of Industrial Ergonomics, vol. 38, no. 5-6, pp. 368-383, 2008.

[24] X. Zhang, "Measurement and modelling of seating dynamics to predict seat transmissibility," Ph.D. Dissertation, University of Southampton, Southampton, England, 2014.

[25] X. Zhang, Y. Qiu, and M. J. Griffin, "Transmission of vertical vibration through a seat: effect of thickness of foam cushions at the seat pan and the backrest," International Journal of Industrial Ergonomics, vol. 48, pp. 36-45, 2015. 